\title{
The cystic fibrosis transmembrane conductance regulator as a biomarker in non-small cell lung cancer
}

\author{
JIN LI $^{1 *}$, JIE TING ZHANG $^{2 *}$, XIAOHUA JIANG ${ }^{2,3^{*}}$, XIAOSHUN SHI $^{1}$, JIANFEI SHEN $^{1}$, FENGLAN FENG $^{1}$, \\ JING YI CHEN ${ }^{1}$, GUIHONG LIU ${ }^{1}$, PING HE ${ }^{1}$, JUHONG JIANG ${ }^{1}$, LAI LING TSANG ${ }^{2}$, YAN WANG ${ }^{2}$, \\ RAFAEL ROSELL ${ }^{5}$, LONG JIANG ${ }^{1}$, JIANXING HE ${ }^{1}$ and HSIAO CHANG CHAN ${ }^{2-4}$ \\ ${ }^{1}$ State Key Laboratory of Respiratory Disease, The First Affiliated Hospital of Guangzhou Medical University, \\ National Clinical Research Center for Respiratory Disease, Guangzhou, Guangdong; ${ }^{2}$ Epithelial Cell Biology \\ Research Center, School of Biomedical Sciences, Faculty of Medicine, The Chinese University of Hong Kong, Shatin, \\ Hong Kong; ${ }^{3}$ Key Laboratory for Regenerative Medicine (Jinan University-CUHK), Ministry of Education, Hong Kong, \\ SAR; ${ }^{4}$ Sichuan University-The Chinese University of Hong Kong Joint Laboratory for Reproductive Medicine, \\ West China Second University Hospital, Sichuan University, Chengdu, Sichuan, P.R. China; \\ ${ }^{5}$ Catalan Institute of Oncology, Badalona, Catalonia, Spain
}

Received November 16, 2014; Accepted January 12, 2015

DOI: 10.3892/ijo.2015.2921

\begin{abstract}
An increased risk of non-small cell lung cancer (NSCLC) in cystic fibrosis (CF) patients and carriers of CF transmembrane conductance regulator (CFTR) mutations has been proposed. However, the role of CFTR in lung cancer remains controversial. In the present study, CFTR expression was assessed in 165 NSCLC tumors and 22 normal lung samples with validation in an independent series of 131 samples. The effect of gain and loss of CFTR on the malignant behavior of NSCLC was examined. The effect of CFTR manipulation on tumor metastasis was examined in a mouse model. Expression of CFTR was downregulated in NSCLC $(\mathrm{P}=0.041)$. Low CFTR expression was correlated with advanced stage $(\mathrm{P}<0.001)$ and lymph node metastasis $(\mathrm{P}=0.009)$. Low $\mathrm{CFTR}$ expression was significantly associated with poor prognosis (overall survival: 45 vs. 36 months, $\mathrm{P}<0.0001$; progression-free survival: 41 vs. 30 months, $\mathrm{P}=0.007)$. Knockdown of CFTR in NSCLC cells enhanced malignant behavior (epithelial-mesenchymal transition, invasion and migration); in contrast, overexpression of CFTR suppressed cancer progression in vitro and in vivo.
\end{abstract}

Correspondence to: Dr Jianxing He, State Key Laboratory of Respiratory Disease, The First Affiliated Hospital of Guangzhou Medical University, National Clinical Research Center for Respiratory Disease, Guangzhou, Guangdong, P.R. China

E-mail: hejx@vip.163.com

Dr Hsiao Chang Chan, Epithelial Cell Biology Research Center, School of Biomedical Sciences, Faculty of Medicine, The Chinese University of Hong Kong, Shatin, Hong Kong, SAR, P.R. China

E-mail: hsiaocchan@cuhk.edu.hk

*Contributed equally

Key words: lung cancer, cystic fibrosis transmembrane conductance regulator, epithelial-mesenchymal transition, prognosis
The tumor-suppressing effect of CFTR was associated with inhibition of multiple uPA/uPAR-mediated malignant traits in culture. These results show that CFTR plays a role in inhibition of NSCLC metastasis and suggest that CFTR may serve as a novel indicator for predicting adverse prognosis and metastasis in NSCLC patients.

\section{Introduction}

Non-small cell lung cancer (NSCLC) is one of the most common malignancies with increasing incidence worldwide (1-3). Despite the advances in early detection and improvements in the treatment, long-term survival from NSCLC still remains poor. Tumor relapse and metastasis are the main factors influencing patient prognosis. The identification of biomarkers that can predict the risk of recurrence and metastasis is therefore clinically important.

The cystic fibrosis transmembrane conductance regulator (CFTR) is a cAMP-activated anion channel which is expressed ubiquitously in epithelial tissues. Germline mutations in the gene encoding CFTR cause recessive cystic fibrosis (CF) (4,5). Over the last three decades, long-term survival rate for $\mathrm{CF}$ patients has significantly improved but an elevated risk of cancer is being recognized to be associated with survivorship. Intriguingly, an increased risk of cancer, primarily of the gastrointestinal tract, has been reported in some, but not all studies in the carriers of CFTR mutations (6-10). Hypermethylation of the CFTR promoter is frequently seen in a number of different tumor types, including lung cancer (11-13), suggesting DNA methylationmediated transcription silencing of CFTR may influence cancer development $(9,14,15)$. Of note, both mutation and hypermethylation of CFTR have been identified in NSCLC patients $(12,16)$.

By analyzing a series of tumors from 296 lung cancer patients we have shown that aberrant CFTR expression level is significantly associated with NSCLC progression, metastasis and poor prognosis. We have also shown that suppression of 
CFTR promotes epithelial-mesenchymal transition (EMT) and metastasis providing mechanistic basis for the role of CFTR in lung cancer.

\section{Materials and methods}

Sample accrual. All samples were collected during surgery at the Department of Surgery, the First Affiliated Hospital of Guangzhou Medical University. One hundred and sixty-five tumor samples and 22 normal lung tissue were obtained from patients enrolled between April 2007 and June 2009. An additional 131 tumor samples were collected from patients recruited for the validation phase during the period between June 2009 and June 2010. Tissue was snap-frozen in liquid nitrogen and stored at $-80^{\circ} \mathrm{C}$. Diagnosis of lung cancer was confirmed at the time of diagnosis after surgery and the presence of tumor cells was verified by a pathologist (G.H.L.) using $\mathrm{H} \& \mathrm{E}$ stained frozen sections in accordance with the World Health Organization guidelines (17). Tumor stage was assigned according to the American Joint Committee for Cancer criteria (18). The background samples were confirmed free of tumor deposits. Clinical follow-up data on patients were ascertained by review of medical records. The follow-up time was up to 60 months. All human specimens and correlative data were obtained according to a protocol reviewed and approved by the local ethics committee, and all patients provided signed informed consent.

Expression analysis of CFTR by real-time PCR and immunochemical staining. Real-time reverse-transcriptase polymerase chain reactions (RT-PCRs) with patient-derived cDNA were performed on a StepOne Plus real-time PCR system (Applied Biosystems, Forster City, USA). Briefly, RNA isolation was carried out using TRI reagent from Sigma (St. Louis, MO, USA), according to the manufacturer's instructions. RNA $(0.5 \mu \mathrm{g})$ was converted into cDNA using the iScript ${ }^{\mathrm{TM}}$ cDNA Synthesis kit (Bio-Rad Laboratories, Hemel Hemstead, UK). The levels of CFTR mRNA were quantified by TaqMan Gene Expression Assays (Assay ID CFTR: Hs00357004_m1; Applied Biosystems) using GAPDH as the internal control. All assays were performed in triplicate. CFTR expression levels were dichotomized by median values.

The tissue blocks were cut into 5-mm sections and processed for IHC in accordance with a previously described protocol (19).

Statistical analysis. Progression-free survival (PFS) was defined as the minimum interval from the date of diagnosis to the date of tumor recurrence, progression, the occurrence of a second malignancy, death or the last follow-up. Overall survival (OS) was defined as the interval from the date of diagnosis to the date of death or the last follow-up. Living patients with local recurrence or metastasis were considered as 'in disease survival'.

The correlations between the CFTR levels and other demographical and clinical features were assessed using Mann-Whitney rank sum and Kruskal-Wallis one way analysis of variance tests.

Patients were assigned to two equal sized groups as defined by the median CFTR level. Kaplan-Meier survival curves and log-rank tests were used to evaluate the differences in OS and PFS between patients groups. Cox proportional hazards model was used to estimate hazard ratios (HRs) and 95\% CIs of CFTR levels at the presence of other demographical and clinical parameters.

Survival analyses were carried out using the SPSS statistical software (version 11; SPSS, Inc., Chicago, IL, USA). All statistical tests were two-sided and any tests with P-value $<0.05$ are considered statistically significant.

Cell lines, antibodies and reagents. The human lung adenocarcinoma cell lines A-549 and H1299 were obtained from the American Type Culture Collection (ATCC, Manassas, VA, USA). Antibodies were used against the following: CFTR (Almone labs, Jerusalem, Israel); E-cadherin, uPA, uPAR and vimentin (Santa Cruz Biotechnology, Santa Cruz, CA, USA); GAPDH (Santa Cruz Biotechnology); CFTRinh-172 (Sigma).

Functional studies. CFTR channel function was blocked by CFTRinh-172 $(10 \mu \mathrm{M})$. For wound healing assay, $1 \times 10^{6}$ cells/well cells were seeded in 6-well plates and then pre-incubated for $24 \mathrm{~h}$ before creating a 'wound' for the cell monolayer with a plastic tip. Cells were then grown in culture medium with $1 \%$ FBS in the presence or absence of $10 \mu \mathrm{M}$ inh-172. The migration of cells was tracked and recorded using a Time-lapse imaging system (Carl Zwiss) for $48 \mathrm{~h}$. Cell migration was determined by measuring distances between parallel lines from initial sites to migrated sites. The procedure was repeated three times. Cell invasion assay was performed using modified Boyden chambers $(8-\mu \mathrm{m})$, polycarbonate membranes (Corning Incorp.) coated with $500 \mu \mathrm{g} / \mathrm{ml}$ Matrigel (BD Biosciences, San Jose, CA, USA). A total of 20,000 cells were added to the Transwell inserts over the top of the artificial basement membrane. Cell growth rate was measured with an MTS proliferation assay. The CellTiter 96 Aqueous One Solution Cell Proliferation assay (Promega) was conducted according to the manufacturer's instructions.

Western blot analysis. Whole-cell extraction was conducted using RIPA buffer [50 mM Tris, $150 \mathrm{mM} \mathrm{NaCl}, 1 \%$ Triton $\mathrm{X}-100,0.1 \%$ SDS and $1 \%$ nadeoxycholate (pH 7.4)] supplemented with protease inhibitors, phenylmethylsulfonyl fluoride and pimix. Protein concentrations were then measured by Bio-Rad protein assay kits (Bio-Rad Laboratories, Hercules, CA, USA). Protein lysates were resolved by SDS-PAGE and then transferred onto nitrocellulose membranes (Hybond ${ }^{\mathrm{TM}}-\mathrm{P}$; Amersham Biosciences, Piscataway, NJ, USA), blocked with TBS containing $0.2 \%$ Tween-20 (TBST) and 5\% non-fat dry milk and incubated with primary antibodies at $4^{\circ} \mathrm{C}$ overnight. Antibody to GAPDH was used as control for protein loading. After being washed with TBST, membranes were incubated with secondary antibody in room temperature for $1 \mathrm{~h}$ before exposure.

CFTR overexpression and knockdown. The pEGFPC3 plasmid expressing wild-type CFTR was provided by Professor Tzyh-Chang Hwang (University of Missouri-Columbia). For overexpression experiments, the A-549 cells were transfected with $3 \mu \mathrm{g}$ DNA and $6 \mu$ l Lipofectamine 2000 (Invitrogen, Camarillo, CA, USA). The transfected cells were selected 
in full medium containing G418 (Calbiochem, Schwalbach, Germany) at 1,200 $\mu \mathrm{g} / \mathrm{ml}$. To knock down CFTR expression, duplex specific miRNAs to human CFTR was synthesized by utilizing Lift Technologies. The siRNA sequence used was: 5'-TTG GAA AGG AGA CTA ACA AG-3'. MiR expression vector, named as pcDNA ${ }^{\mathrm{TM}} 6.2-\mathrm{GW} / \mathrm{EmGFP}$, containing a double-stranded oligonucleotide (ds-oligo) encoding a pre-miRNA sequence were established using BLOCK-iT ${ }^{\mathrm{TM}}$ Pol II miR RNAi Expression Vector kits (Life Technologies, Rockville, MD, USA) following the manufacturer's protocol. Lentiviral particles were produced by transient transfection of 293FT (Invitrogen) cells using Lipofectamine 2000 (Invitrogen) reagent. Blasticidin at the final concentration of $5 \mu \mathrm{g} / \mathrm{ml}$ was used to select the stable clones.

Animal studies. Nude mice were provided by the Laboratory Animal Service Center of the Chinese University of Hong Kong. Animals were properly maintained in an airconditioned room with controlled temperature of $24 \pm 2^{\circ} \mathrm{C}$ and humidity of $55 \pm 15 \%$, in a 12 -h light/dark cycle and were fed laboratory chow and water ad libitum. All animal experiments were conducted in strict accordance with the University Laboratory Animals Service Center guidelines on animal experimentation with approval from the Animal Ethics Committee of the University. Tumorigenicity was investigated by tumor xenograft experiments. Briefly, female athymic balb/c nude mice, 6-8 weeks of age, were injected with $10 \mu \mathrm{l}$ suspension of CFTR knockdown cells or vector control A-549 cells $\left(\sim 5 \times 10^{6}\right)$ subcutaneously. Mice injected with saline were used as sham controls. Tumor formation in nude mice was monitored over approximately a 6 -week period and ratios of tumor weight to body weight were sacrificed. Tumor size, animal health and behavior were measured and monitored twice every week. Mice with a tumor size $>1 \mathrm{~cm}$ in any dimension were terminated. The tumor size was calculated according to the following formula: $0.5234 \mathrm{X}$ [long diameter (short diameter) $\left.{ }^{2}\right]$. To develop the metastatic model, 6-8-week old female balb/c nude mice were transplanted with $1 \times 10^{6}$ A-549 cells through the lateral tail vein under sterile conditions. All mice were sacrificed 6 weeks after injection by perfusion. Before the perfusion surgery, $100 \mathrm{mg} / \mathrm{kg}$ ketamine mixed with $10 \mathrm{mg} / \mathrm{kg}$ xylazine was used. Cold PBS $(10 \mathrm{ml})$ for each mice was perfused through right ventricle until lungs cleared of blood, then $10 \mathrm{ml}$ of cold PFA was perfused to fix the tissue. Lungs were collected, and further fixed in PFA overnight, and sectioned $(5 \mu \mathrm{m})$ in preparation for $\mathrm{H} \& \mathrm{E}$ staining for morphological investigation. Number and size of tumor loci was countered and calculated.

\section{Results}

Downregulation of CFTR expression in NSCLC. A statistically significant lower CFTR transcript level was observed in NSCLC tumor tissues than that in normal lung tissues (Fig. 1A and Table I). Compared to other various NSCLC subtypes, the level of CFTR was significantly higher in adenocarcinoma patients $(\mathrm{P}=0.004$; Fig. 1B). There were no significant differences between patients with high and low CFTR expression levels as determined by age, gender or smoking status (Table I).
Table I. CFTR expression and patient characteristics.

\begin{tabular}{|c|c|c|}
\hline & Samples & CFTR \\
\hline Characteristics & $\begin{array}{c}\mathrm{N} \\
(\%)\end{array}$ & $\begin{array}{l}\text { Median } \\
\left(\text { range }^{\mathrm{a}}\right)\end{array}$ \\
\hline
\end{tabular}

\section{Tissue type \\ Tumor \\ Normal \\ Age (years) \\ $\leq 65$ \\ $>65$ \\ Gender \\ Female \\ Male}

Smoking status

Yes

No

Histology

ADC

SCC

Other

Stage

I

II

IIIA

IIIB

IV

Differentiation

High

Low

Tumor nodularity

Unilateral pulmonary

Bilateral pulmonary

Tumor size $(\mathrm{cm})$

$\leq 2$

$2-5$

$>5$

Pleura involvement

Yes

No

Lymph node involvement

Yes

No

Vascular invasion

Yes

No

Clinical outcome

Alive
Death
Time of survival (months)
$\geq 36$
$<36$

165 (88.2) $0.12(0.55) \quad 0.04$

$22(11.8) \quad 0.42(1.11)$

$115(61.5) \quad 0.09(0.44) \quad 0.14$

49 (26.2) $0.19(0.87)$

$64(34.2) \quad 0.13(0.90)$

0.2

$102(54.5) \quad 0.11(0.36)$

$58(31.0) \quad 0.09(0.32)$

0.12

102 (54.5) $0.13(0.88)$

$139(74.3) \quad 0.15(0.69)$

0.004

$17(9.1) \quad 0.01(0.11)$

9 (4.8) $0.01(1.72)$

53 (28.3) $0.36(1.36)<0.0001$

46 (24.6) $0.11(0.61)$

33 (17.6) $0.08(0.27)$

9 (4.9)

$24(12.8) \quad 0.00(0.02)$

$117(62.6) \quad 0.12(0.84)$

0.95

$38(20.3) \quad 0.14(0.28)$

$158(84.5) \quad 0.13(0.68)$

0.08

$7(3.7) \quad 0.01(0.14)$

$27(14.4) \quad 0.30(0.83)$

0.09

97 (51.9) $0.13(0.61)$

$41(21.9) \quad 0.04(0.20)$

$88(47.1) \quad 0.09(0.44)$

0.3

77 (41.2) $0.13(0.82)$

$92(49.2) \quad 0.06(0.31) \quad 0.01$

73 (39.0) $\quad 0.20(0.94)$

$6(3.2) \quad 0.01(1.71) \quad 0.3$

$159(85.0) \quad 0.13(0.58)$

109 (58.3) $0.14(0.66)$

0.42

$54(28.9) \quad 0.05(0.46)$

$94(50.3) \quad 0.16(0.68)$

0.04

69 (36.9) $0.04(0.47)$ anterquartile range; ADC, adenocarcinoma; SCC, squamous cell carcinoma. 


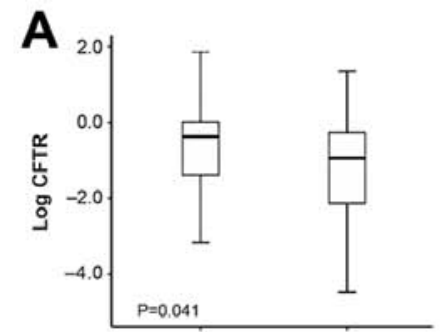

Normal Tumor
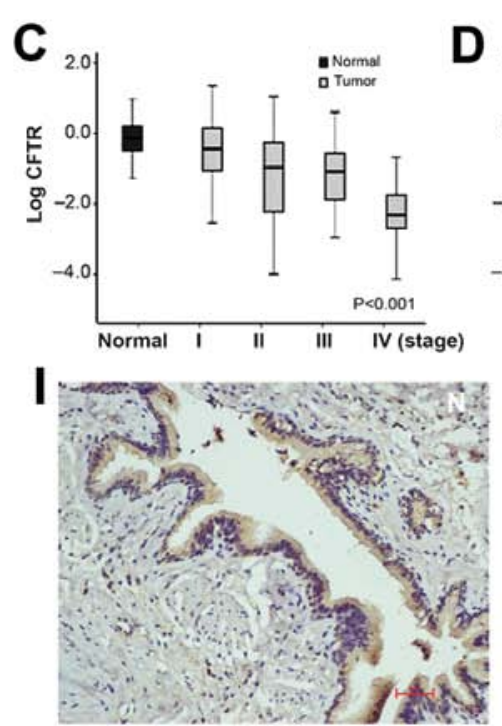

B

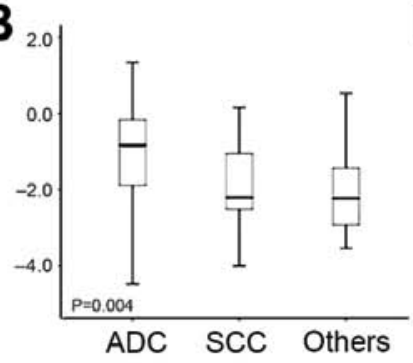

E

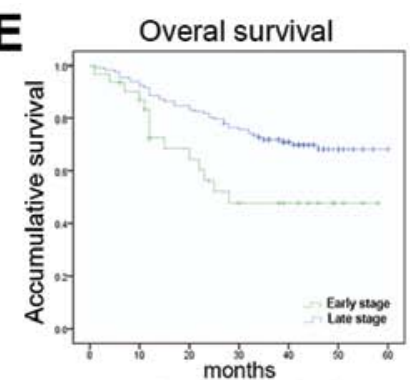

G
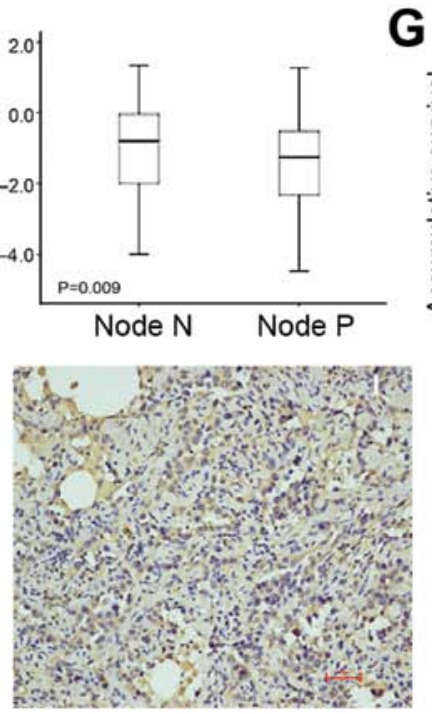
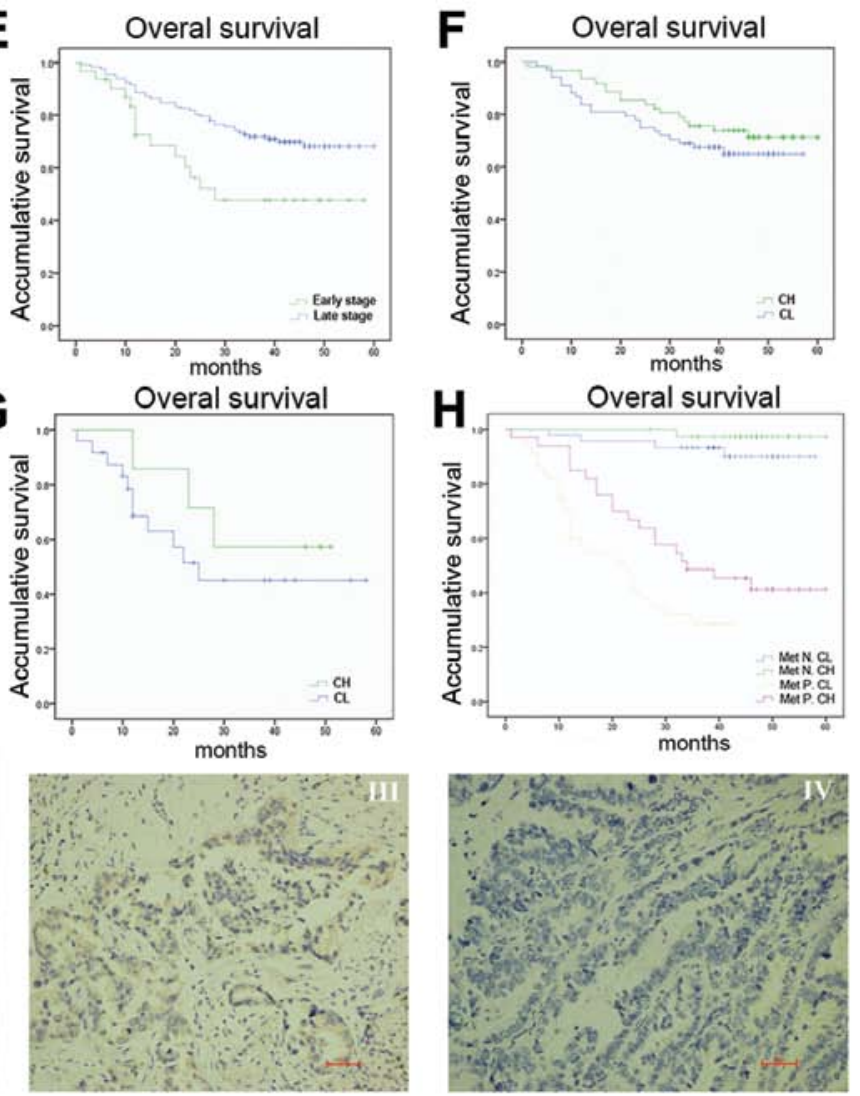

Figure 1. Expression of CFTR in NSCLC and its association with disease progression and prognosis. CFTR mRNA expression level (A) in tumor tissues and normal tissues; (B) in adenocarcinoma, squamous cell carcinoma and other histology types; (C) in different clinical stage progressions and (D) lymph node metastasis. Mann-Whitney test was used to test the difference of CFTR levels across strata. Kaplan-Meier survival curves for (E) early- and late-stage patients; (F) patients groups stratified by dichotomized CFTR expression levels at early-stage and (G) late-stage of prognosis; and (H) patients groups stratified by metastasis status and dichotomized CFTR levels. Met N. CH, group of patients with high CFTR level and without metastasis; Met N. CL, group of patients with low CFTR level and without metastasis; Met P. CH, group of patients with metastasis but with high CFTR level; Met P. CL, group of patients with low CFTR level and with metastasis. (I) Representative images showed high expression of CFTR by IHC in normal lung tissues (left, N), relatively strong staining of CFTR in tumors with stage I (middle left, I) and low expression of CFTR in tumors with stage III (middle right, III) and negative expression of CFTR in stage IV tumor tissues (right, IV) (scale bar, $100 \mu \mathrm{m}$ ).

Lower CFTR expression is associated with advanced disease in NSCLC. Those patients with low CFTR levels in general had more advanced tumors. A gradual decrease in CFTR transcripts levels was observed through stage I to IV patients (Fig. 1C and Table I). The protein levels of CFTR were downregulated in advanced stage tumors compared to normal tissues as assessed by immunohistochemistry (IHC) staining through a panel of cohorts (Fig. 1I). A significantly lower CFTR expression level was also found in patients with lymph node metastasis (node P) compared to lymph node-free patients (node N) (Fig. 1D and Table I). There was evidence of downregulation of CFTR levels in patients with either larger tumor, tumors with poorer differentiation, vascular or pleural invasion, however, this difference was not statistically significant.

Low CFTR gene expression is correlated with poor prognosis and inferior survival. As CFTR expression appeared to be related to disease progression, we analyzed its relationship with prognosis.

The association between CFTR expression levels and disease progression was initially tested in 165 NSCLC patients recruited to the discovery phase. Survival rates were compared between patients groups defined by the median CFTR expression level (0.15). Higher OS (median, 45; 95\% CI, 42.9-47.0) and PFS (median, 41; 95\% CI, 33.8-48.2) were observed in the patients with high CFTR expression level (Fig. 1; P<0.01), compared to lower OS (median, 36; 95\% CI, 32.7-39.3) and PFS (median, 30; 95\% CI, 17.1-42.9). The effect of CFTR expression remained highly significant after adjusted for age, gender, smoking status and clinical stage, compatible with CFTR expression levels being an independent prognosis predictor of clinical stages (Table II). The association between CFTR levels and prognosis were then analyzed against early stage (stage I to IIIA) and late stage (stage IIIB to IV) cancer patients. Although the clinical stage predicted favorable OS (Fig. 1E), patients with high CFTR levels $(\mathrm{CH})$ had significantly better survival regardless of early/late stage disease (Fig. $1 \mathrm{~F}$ and $\mathrm{G}$ ).

The association between CFTR levels and prognosis was then validated in 131 NSCLC patients recruited between 2009-2010. The Kaplan-Meier survival and log-rank tests provided evidence of improved OS for patients with higher CFTR levels albeit not statistically significant.

Pooling the data from discovery and the validation phase using an inverse variance meta-analysis approach showed 
Table II. Cox proportional hazard model.

\begin{tabular}{|c|c|c|c|c|c|c|c|c|c|c|}
\hline \multirow[b]{2}{*}{ Factors } & \multicolumn{3}{|c|}{ Discovery } & \multicolumn{3}{|c|}{ Replication } & \multicolumn{4}{|c|}{ Fixed effect meta-analysis } \\
\hline & HR & $95 \% \mathrm{CI}$ & P-value & HR & $95 \% \mathrm{CI}$ & P-value & HR & $95 \% \mathrm{CI}$ & P-value & $\mathrm{P}_{\text {het }}$ \\
\hline CFTR & 0.52 & $0.37-0.72$ & $<0.001$ & 0.66 & $0.48-0.91$ & 0.01 & 0.69 & $0.62-0.76$ & $3.53 \times 10^{-13}$ & 0.28 \\
\hline Age & 1.24 & $0.87-1.76$ & 0.23 & 1.09 & $0.46-2.58$ & 0.85 & 0.91 & $0.79-1.04$ & 0.17 & 0.18 \\
\hline Gender & 1.11 & $0.74-1.66$ & 0.61 & 1.37 & $0.77-2.44$ & 0.29 & 0.88 & $0.76-1.02$ & 0.09 & 0.92 \\
\hline Smoking status & 0.86 & $0.56-1.31$ & 0.48 & 1.42 & $0.79-2.56$ & 0.25 & 0.82 & 0.71-0.95 & 0.01 & 0.37 \\
\hline Clinical stage & 1.21 & $1.03-1.42$ & 0.02 & 1.06 & $0.74-1.52$ & 0.76 & 0.99 & $0.93-1.05$ & 0.74 & 0.10 \\
\hline
\end{tabular}

Table III. Log-rank test on OS for CFTR with metastasis.

\begin{tabular}{lcccc}
\hline Group & $\begin{array}{c}\text { Patients } \\
(\mathrm{n})\end{array}$ & $\begin{array}{c}\text { Median OS } \\
\text { (months) }\end{array}$ & $95 \%$ CI & P-value \\
\hline Metastasis (-) & & & & \\
Low CFTR & 44 & 54 & $51.3-57.9$ & $<0.0001$ \\
High CFTR & 50 & 58 & $57.0-60.4$ & \\
Total & 94 & 57 & $55.7-59.5$ & \\
Metastasis (+) & & & & \\
Low CFTR & 49 & 24 & $20.6-29.4$ & \\
High CFTR & 44 & 41 & $35.3-47.5$ & \\
Total & 93 & 35 & $30.9-40.0$ & \\
Overall & 187 & 47 & $43.9-49.8$ & \\
\hline
\end{tabular}

a significant association between CFTR level and prognosis independent of clinical stages (HR, 0.69; 95\% CI, 0.62-0.76; $\mathrm{P}=3.53 \times 10^{-13}$ ) (Table II).

Notably four stage IV patients from both phases who remained progression-free showed significantly higher CFTR levels (mean \pm SEM, 4.758 \pm 1.461 ) in comparison to those patients with local recurrence, metastasis or those who died from lung cancer (mean \pm SEM, 0.0289 $\pm 0.754 ; \mathrm{P}=0.003$ ). The surviving stage IV patients had higher CFTR levels (1.611 \pm 0.863$)$, compared to those who died of the disease $(0.030 \pm 0.996)$, indicated a particular role of CFTR in late stage prognosis.

We analyzed OS stratified by CFTR expression levels and metastasis status. Median OS rates were computed and compared among the patient groups using log-rank testing (Fig. 1H). For patients without metastasis, the median survival of high CFTR level group (Met N. CH) was 58 months while that of the low CFTR level group (Met N. CL) was 54 months. For the patients with metastasis, patients with high CFTR levels (Met P. CH) experienced a considerably longer 41-month median survival compared with the 24-month median survival of the low CFTR expression group (Met P. CL) (Table III).

Manipulation of CFTR alters malignancy of lung cancer in vitro and in vivo. The observed association between CFTR expression level and NSCLC metastasis and prognosis prompted us to investigate whether CFTR gene manipulation might affect the malignant phenotype. The alteration of CFTR expression did not significantly affect proliferation of NSCLC cell line A-549 (data not shown). However, cell invasion and migration were significantly enhanced or suppressed by knockdown or overexpression of CFTR, respectively (Fig. 2B, C, E and F). The same trend was seen in H1299 cells (data not shown).

To examine the role of CFTR in tumorigenicity in vivo, we established xenograft models by subcutaneous injection of A-549 cells transduced with shRNA targeting CFTR in mice. No differences in primary tumor growth between control and CFTR-knockdown A-549-injected mice were seen (data not shown), however, $80 \%$ (4/5) of the mice injected with CFTRknockdown cells presented with lung metastasis in contrast to $40 \%(2 / 5)$ of the mice injected with A-549-empty controls (Fig. 2G and H). Moreover, the tumor burden in the vector and CFTR knockdown groups were significantly different 6 weeks after the tumor cell inoculation (Fig. 2I).

CFTR regulates EMT through the UPA/UPAR pathway. Application of the CFTR activity inhibitor, inh-172 led A-549 cells to rapidly change their morphology into an elongated fibroblast-like shape (Fig. 3A). This was reflected in downregulation of epithelial marker expression including E-cadherin and upregulated mesenchymal markers such as vimentin (Vim) and fibronectin (Fig. 3B), suggesting suppression of CFTR function promotes EMT in lung cancer. The effect of CFTR on EMT in lung cancer cells was also supported by the observation that CFTR knockdown promoted EMT, whereas CFTR overexpression inhibited EMT (Fig. 3C and D). It has been reported that CFTR is involved in the NF- $\kappa \mathrm{B}$ mediated urokinase-type plasminogen activator (uPA) pathway, which is associated with cancer development (20-23). Given the central role of the uPA/uPAR axis in EMT (24), we reasoned that CFTR might be implicated in the regulation of EMT and lung cancer metastasis through the uPA/uPAR pathway. As shown in Fig. 4A and B, knockdown of CFTR increased actived-uPA and UPAR protein expression, while overexpression of CFTR inhibited actived-uPA and uPAR expression in A-549 cells (Fig. 4A and B). The activated level of uPA was accordingly altered by CFTR gene manipulation, indicating that UPA activity is CFTR-dependent in these cancer cells. Neutralization with UPA or UPAR antibodies in CFTR-knockdown A-549 cells dramatically reversed the 
A

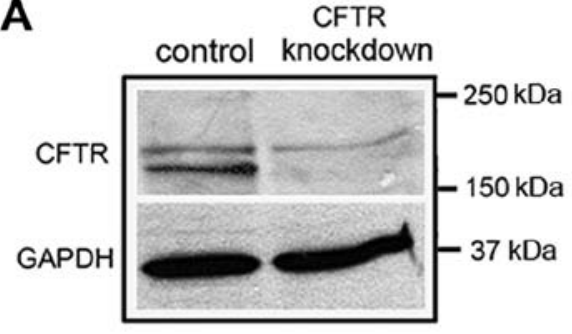

D

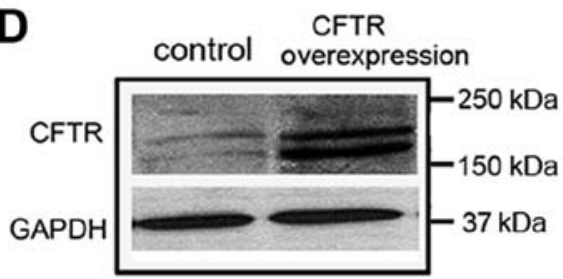

B

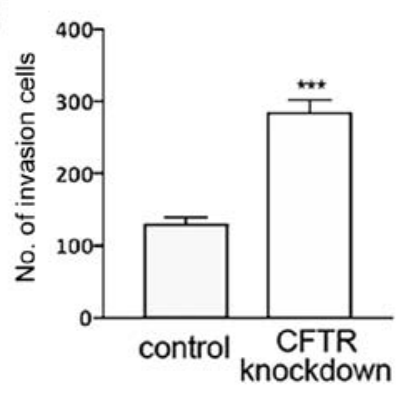

E

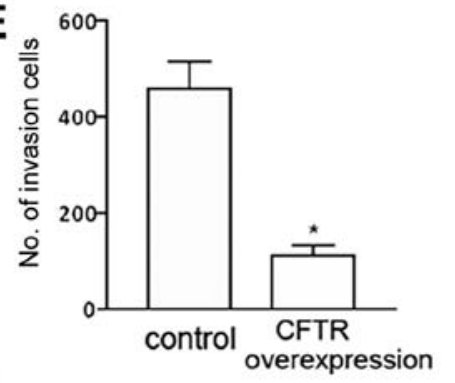

C

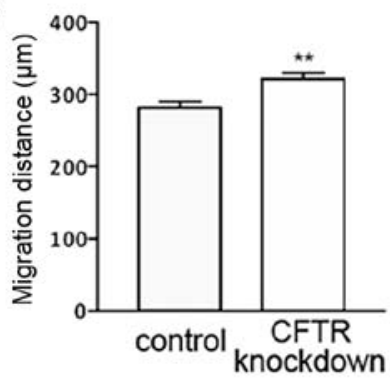

F

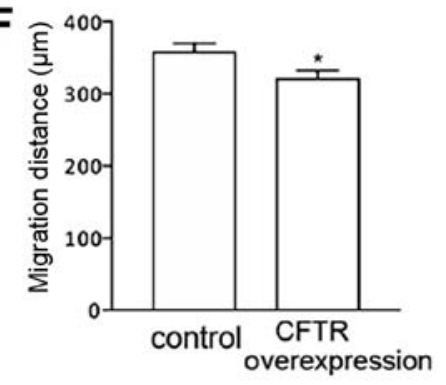

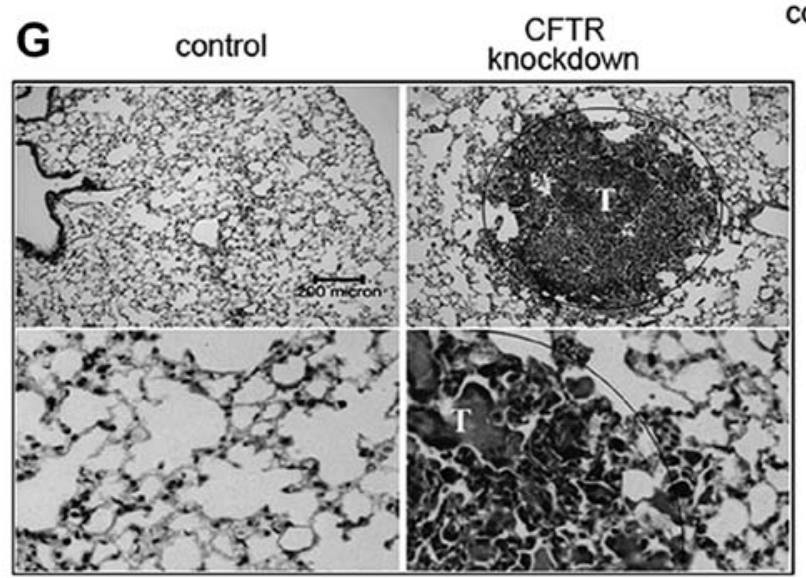

H

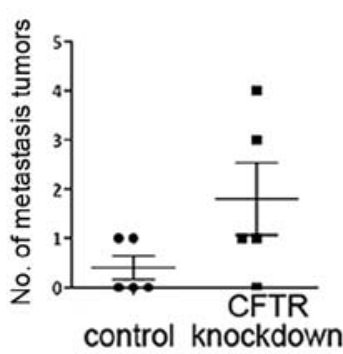

I

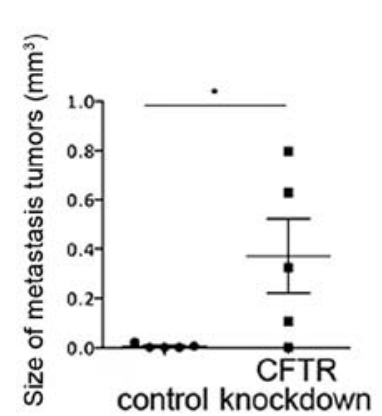

Figure 2. CFTR gene manipulation alters metastasis of lung cancer in vitro and in vivo. (A) Western blot analysis showing that the expression of CFTR is significantly repressed by miR-CFTR comparing to control in A-549 cells. Both mature and immature bands of CFTR can be detected in A-549 with a molecular weight $>150 \mathrm{kD}$. Image is representative of three independent experiments. (B) CFTR-knockdown A-549 cells show enhanced cell invasion ability comparing to control cells in $48 \mathrm{~h} ;{ }^{* * *} \mathrm{P}<0.001$. (C) Wound healing assay shows migration ability is enhanced in CFTR-knockdown A-549 cells; ${ }^{* *} \mathrm{P}<0.01$. (D) Western blot analysis showing that the expression of CFTR in control and CFTR-overexpressing A-549 cells. Image is representative of three independent experiments. (E) Overexpression of CFTR represses cell invasion in CFTR-overexpressing A-549 cells comparing to control cells after 72 h; * $\mathrm{P}<0.05$. (F) Cell migration in A-549 cells is significantly repressed by overexpression of CFTR; ${ }^{\mathrm{P}}<0.05$. (G-I) H\&E staining of the lung in mice 6 weeks after injection with A-549 cells transfected with control or microRNA target CFTR. (G) Representative image showing tumor loci in mouse lungs injected with miR-CFTR-transduced A-549 cells. Tumor area is circled and labeled as 'T'. (H) Mice injected with CFTR knockdown cells show more metastatic loci comparing to mice injected with control cells, ${ }^{*} \mathrm{P}<0.05$. (I) Metastatic tumor size is significantly larger in CFTR knockdown mice comparing to control mice; ${ }^{*} \mathrm{P}<0.05$.

CFTR knockdown-enhanced cell migration and invasion in these cells (Fig. 4C and D), indicating that upregulation of the uPA/uPAR axis activity is the major mechanism leading to the observed increased malignancies induced by CFTR knockdown.

\section{Discussion}

The recent discoveries of genomic alterations, including mutation of EGFR, KRAS and ALK genes in NSCLC, have suggested novel therapeutic targets for the treatment of lung cancer (25-28). A biomarker for the prediction of NSCLC recurrence and metastasis, however, remains to be identified.

In the present study, CFTR was significantly downregulated in NSCLC tumors and low expression of CFTR was significantly correlated with NSCLC progression and metas- tasis suggesting CFTR as a prognostic biomarker for lung cancer. Importantly, our findings are consistent with CFTR having a strong protective influence in NSCLC, especially in the context of late-stage disease.

One of the key processes that occur during the progression of tumor metastasis is EMT. In the present study, the suppression of CFTR function leads to loss of epithelial markers, whereas, overexpression of CFTR results in upregulation of epithelial markers. This is in line with the observed changes of EMT process and invasive phenotypes in lung cancer cell lines, and is consistent with a metastasis-suppressing role of CFTR.

The present study also demonstrated that CFTR can suppress lung cancer metastasis through the uPA system. The uPA system has a multifunctional role in neoplastic evolution, affecting cancer cell proliferation, tumor angiogenesis, 
A

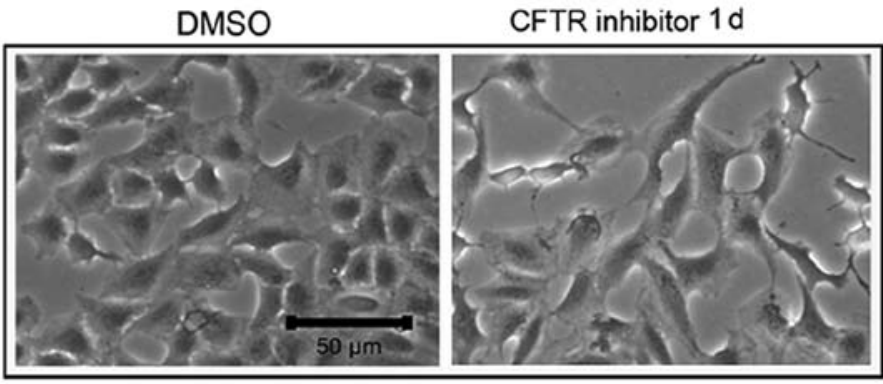

B
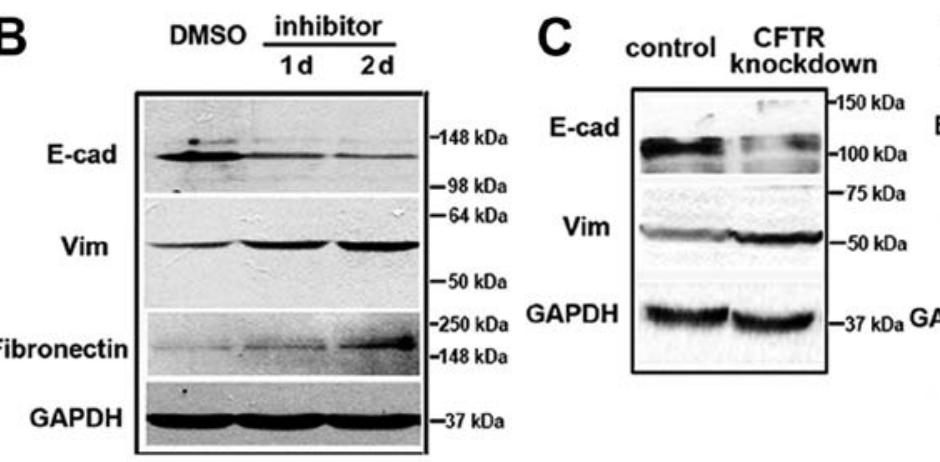

D control CFTR

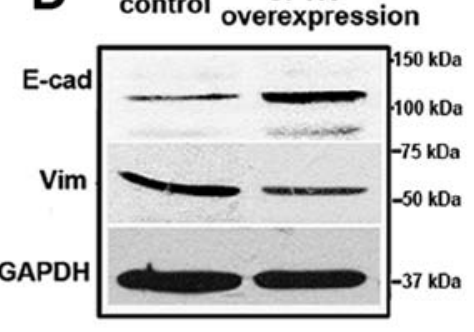

Figure 3. CFTR is associated with EMT. A-549 cells were treated with $10 \mu \mathrm{M}$ inh-172 for 24 and $48 \mathrm{~h}$. (A) Phase-contrast images show that inhibition of CFTR alters the cellular morphology to elongated-fibroblast like cells after inh-172 treatment for $24 \mathrm{~h}$ (scale bar, $50 \mu \mathrm{m}$ ). (B) Western blot analysis shows CFTR inhibitor induces upregulation of the mesenchymal markers vimentin and fibronectin and downregulation of the epithelial marker E-cadherin (precursor, $135 \mathrm{kDa}$; mature E-cadherin, $120 \mathrm{kDa}$ ). (C) Western blot analysis showing epithelial marker E-cadherin is downregulated whereas vimentin expression is upregulated in A-549 with CFTR knockdown. (D) Western blot analysis showing epithelial marker E-cadherin is upregulated in A-549 with CFTR overexpression. Image is representative of three independent experiments.

A

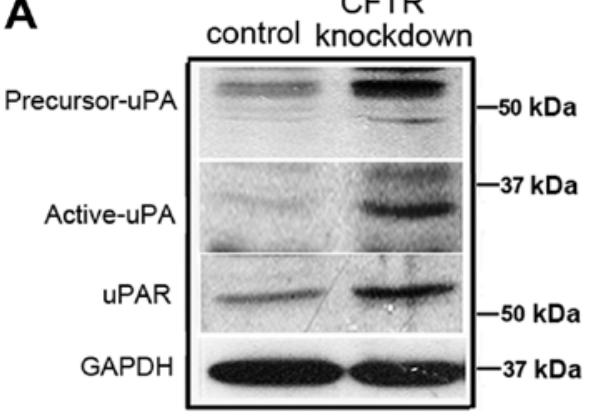

C

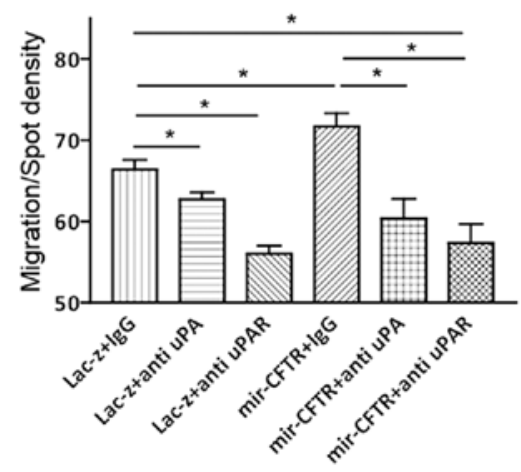

B

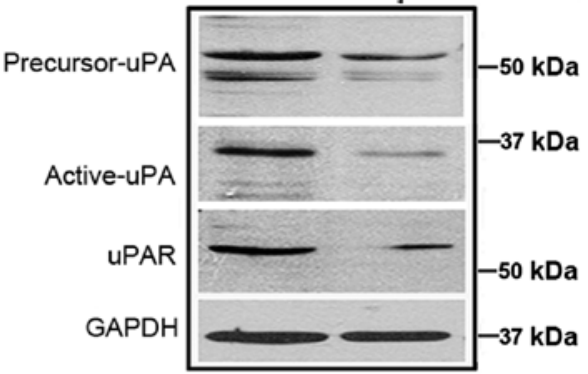

D

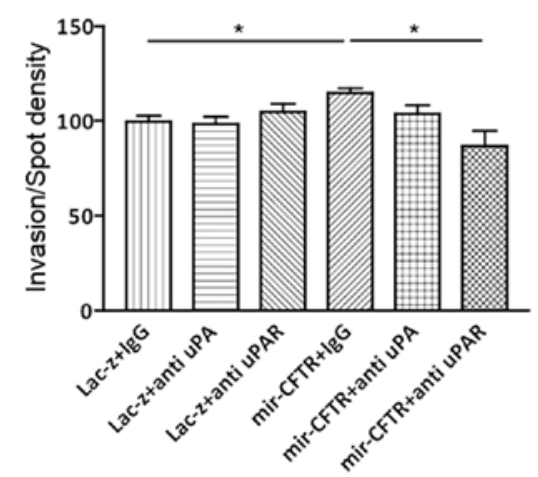

Figure 4. Involvement of uPA pathway in the migration-suppressing effects of CFTR. Western blots results show that the expression of active-uPA and uPAR is (A) upregulated in A-549 CFTR knockdown and (B) downregulated in CFTR-overexpressing A-549 cells vs. vector control cells. Image is representative of three independent experiments. (C and D) Using uPA and uPAR antibody block uPA activity, CFTR-knockdown induced (C) migration and (D) invasion were reversed in A-549 cells; $\left({ }^{*} \mathrm{P}<0.05\right)$.

adhesion and migration (24). Furthermore, increased expression of UPA, UPAR and PAI-1 has been documented in many types of cancers (29). In the present study, uPA expression was inversely associated with CFTR expression in lung 
cancer cells. In addition, uPA or UPAR neutralizing antibody reversed the enhanced cell migration and invasion of CFTR knockdown. It is well established that CFTR is a negative regulator of $\mathrm{NF}-\kappa \mathrm{B}(21-23)$, and that $\mathrm{NF}-\kappa \mathrm{B}$ positively regulates uPA during cancer development and progression $(30,31)$. Therefore, it is possible that the regulation of CFTR on the uPA pathways may be mediated through the negative mediation of NF- $\kappa \mathrm{B}$. Since other EMT-inducing factors, such as TNF $\alpha$ and HIF $1 \alpha$, have been demonstrated to downregulate CFTR expression $(32,33)$ and CFTR regulate EMT through interaction with AF-6 and miR-193b in other cancer $(34,35)$, CFTR may act as a conjoint downstream effector in mediating the effects of EMT inducers.

In the present study, we have provided evidence that CFTR expression may be a biomarker for lung cancer metastasis and prognosis. Moreover, since CFTR may also be a prognostic predictor in breast cancer (36) it suggests that CFTR may have a more generic function in cancer development, both in vitro and in vivo functional studies and in evaluation of CFTR expression. Of note, drugs that target specific CFTR point mutations have shown therapeutic benefit in patients with $\mathrm{CF}$ (37) and naturally occurring polyphenol compound resveratrol may stimulate the activity of CFTR (38). The results of the present study warrant further investigation exploring the utility of these agents in the management of advanced lung cancer.

\section{Acknowledgements}

We are grateful to Professor Tzyh-Chang Hwang of the Department of Biological Engineering, University of Missouri-Columbia, USA, for providing CFTR-peGFPC3 and the control plasmids. The present study was supported in part by National 973 projects (2013CB967403, 2013CB967404), the National Natural Science Foundation of China (81201845), the Guangzhou Medical University (2011A06) and the Research Grant Council of Hong Kong (GRF-CUHK466413) and the Focused Investment Scheme of the Chinese University of Hong Kong.

\section{References}

1. Chang S, Dai M, Ren JS, Chen YH and Guo LW: Estimates and prediction on incidence, mortality and prevalence of lung cancer in China in 2008. Zhonghua Liu Xing Bing Xue Za Zhi 33: 391-394, 2010 (In Chinese).

2. Chien CR and Chen TH: A Bayesian model for age, period, and cohort effects on mortality trends for lung cancer, in association with gender-specific incidence and case-fatality rates. J Thorac Oncol 4: 167-171, 2009.

3. Subramanian J, Madadi AR, Dandona M, Williams K, Morgensztern D and Govindan R: Review of ongoing clinical trials in non-small cell lung cancer: a status report for 2009 from the ClinicalTrials.gov website. J Thorac Oncol 5: 1116-1119, 2010.

4. Collins FS: Cystic fibrosis: molecular biology and therapeutic implications. Science 256: 774-779, 1992.

5. Riordan JR, Rommens JM, Kerem B, et al: Identification of the cystic fibrosis gene: cloning and characterization of complementary DNA. Science 245: 1066-1073, 1989.

6. McWilliams RR, Rabe KG, Olswold C, De Andrade M and Petersen GM: Risk of malignancy in first-degree relatives of patients with pancreatic carcinoma. Cancer 104: 388-394, 2005.

7. McWilliams RR, Petersen GM, Rabe KG, et al: Cystic fibrosis transmembrane conductance regulator (CFTR) gene mutations and risk for pancreatic adenocarcinoma. Cancer 116: 203-209, 2010 .
8. Neglia JP, FitzSimmons SC, Maisonneuve P, et al: The risk of cancer among patients with cystic fibrosis. Cystic Fibrosis and Cancer Study Group. N Engl J Med 332: 494-499, 1995.

9. Li Y, Sun Z, Wu Y, et al: Cystic fibrosis transmembrane conductance regulator gene mutation and lung cancer risk. Lung Cancer 70: 14-21, 2010.

10. Maisonneuve P, Marshall BC, Knapp EA and Lowenfels AB: Cancer risk in cystic fibrosis: a 20-year nationwide study from the United States. J Natl Cancer Inst 105: 122-129, 2013.

11. Mishra DK, Chen Z, Wu Y, Sarkissyan M, Koeffler HP and Vadgama JV: Global methylation pattern of genes in androgensensitive and androgen-independent prostate cancer cells. Mol Cancer Ther 9: 33-45, 2010.

12. Son JW, Kim YJ, Cho HM, et al: Promoter hypermethylation of the CFTR gene and clinical/pathological features associated with non-small cell lung cancer. Respirology 16: 1203-1209, 2011.

13. Ding S, Gong BD, Yu J, et al: Methylation profile of the promoter CpG islands of 14 'drug-resistance' genes in hepatocellular carcinoma. World J Gastroenterol 10: 3433-3440, 2004.

14. Abraham EH, Vos P, Kahn J, et al: Cystic fibrosis hetero- and homozygosity is associated with inhibition of breast cancer growth. Nat Med 2: 593-596, 1996.

15. O'Connell MP, Fiori JL, Baugher KM, et al: Wnt5A activates the calpain-mediated cleavage of filamin A. J Invest Dermatol 129: 1782-1789, 2009.

16. Govindan R, Ding L, Griffith M, et al: Genomic landscape of non-small cell lung cancer in smokers and never-smokers. Cell 150: 1121-1134, 2012.

17. Brambilla E, Travis WD, Colby TV, Corrin B and Shimosato Y: The new World Health Organization classification of lung tumours. Eur Respir J 18: 1059-1068, 2001.

18. Edge SB and Compton CC: The American Joint Committee on Cancer: the 7th edition of the AJCC cancer staging manual and the future of TNM. Ann Surg Oncol 17: 1471-1474, 2010.

19. Li J, Ye L, Mansel RE and Jiang WG: Potential prognostic value of repulsive guidance molecules in breast cancer. Anticancer Res 31: 1703-1711, 2011.

20. Smith SM, Vaughan JM, Donaldson CJ, et al: Cocaine- and amphetamine-regulated transcript is localized in pituitary lactotropes and is regulated during lactation. Endocrinology 147: 1213-1223, 2006.

21. Vij N, Mazur S and Zeitlin PL: CFTR is a negative regulator of NFkappaB mediated innate immune response. PLoS One 4: e4664, 2009.

22. DiMango E, Ratner AJ, Bryan R, Tabibi S and Prince A: Activation of NF-kappaB by adherent Pseudomonas aeruginosa in normal and cystic fibrosis respiratory epithelial cells. J Clin Invest 101: 2598-2605, 1998.

23. Boncoeur E, Roque T, Bonvin E, et al: Cystic fibrosis transmembrane conductance regulator controls lung proteasomal degradation and nuclear factor-kappaB activity in conditions of oxidative stress. Am J Pathol 172: 1184-1194, 2008.

24. Smith HW and Marshall CJ: Regulation of cell signalling by uPAR. Nat Rev Mol Cell Biol 11: 23-36, 2010.

25. Lipson D, Capelletti M, Yelensky R, et al: Identification of new ALK and RET gene fusions from colorectal and lung cancer biopsies. Nat Med 18: 382-384, 2012.

26. Kohno T, Ichikawa H, Totoki Y, et al: KIF5B-RET fusions in lung adenocarcinoma. Nat Med 18: 375-377, 2012.

27. Soda M, Choi YL, Enomoto M, et al: Identification of the transforming EML4-ALK fusion gene in non-small-cell lung cancer. Nature 448: 561-566, 2007.

28. Takeuchi K, Soda M, Togashi Y, et al: RET, ROS1 and ALK fusions in lung cancer. Nat Med 18: 378-381, 2012.

29. Strongin AY: Proteolytic and non-proteolytic roles of membrane type-1 matrix metalloproteinase in malignancy. Biochim Biophys Acta 1803: 133-141, 2010.

30. Lawrence T: The nuclear factor NF-kappaB pathway in inflammation. Cold Spring Harb Perspect Biol 1: a001651, 2009.

31. Sen R and Smale ST: Selectivity of the NF-\{kappa\}B response. Cold Spring Harb Perspect Biol 2: a000257, 2010.

32. Pruliere-Escabasse V, Fanen P, Dazy AC, et al: TGF-beta 1 downregulates CFTR expression and function in nasal polyps of non-CF patients. Am J Physiol Lung Cell Mol Physiol 288: L77-L83, 2005. 
33. Howe KL, Wang A, Hunter MM, Stanton BA and McKay DM: TGFbeta down-regulation of the CFTR: a means to limit epithelial chloride secretion. Exp Cell Res 298: 473-484, 2004.

34. Xie C, Jiang XH, Zhang JT, et al: CFTR suppresses tumor progression through miR-193b targeting urokinase plasminogen activator (uPA) in prostate cancer. Oncogene 32: 2282-2291, 2013.

35. Sun TT, Wang Y, Cheng H, et al: Disrupted interaction between CFTR and AF-6/afadin aggravates malignant phenotypes of colon cancer. Biochim Biophys Acta 1843: 618-628, 2014.
36. Zhang JT, Jiang XH, Xie C, et al: Downregulation of CFTR promotes epithelial-to-mesenchymal transition and is associated with poor prognosis of breast cancer. Biochim Biophys Acta 1833: 2961-2969, 2013.

37. Ramsey BW, Davies J, McElvaney NG, et al: A CFTR potentiator in patients with cystic fibrosis and the G551D mutation. N Engl J Med 365: 1663-1672, 2011.

38. Yang S, Yu BO, Sui Y, et al: CFTR chloride channel is a molecular target of the natural cancer preventive agent resveratrol. Pharmazie 68: 772-776, 2013. 